EPJ manuscript No.

(will be inserted by the editor)

\title{
Hypernuclear Physics and Compact Stars
}

\author{
Jürgen Schaffner-Bielich \\ Institut für Theoretische Physik, J. W. Goethe Universität, D-60438 Frankfurt am Main, Germany \\ Received: date / Revised version: date
}

\begin{abstract}
Hypernuclear physics plays a decisive role for several features of compact star physics. I review the impact of hypernuclear potential depths, two-body hyperon-nucleon and hyperon three-body forces as well as hyperon-hyperon interactions on the maximum mass, the mass-radius relation, and cooling properties of neutron stars.

PACS. 21.80.+a Hypernuclei - 26.60.+c Nuclear matter aspects of neutron stars - 97.60.Jd Neutron stars - 12.39.Mk Glueball and nonstandard multi-quark/gluon states
\end{abstract}

\section{Introduction}

One of the most enigmatic and extreme astrophysical objects are considered to be neutron stars, which are produced in spectacular core collapse supernova explosions. These compact, massive objects have typical radii of about $10 \mathrm{~km}$ and masses of $1-2 M_{\odot}$. Matter in the core of neutron stars experiences extreme densities, several times normal nuclear matter density, i.e. $n \gg n_{0}=3 \cdot 10^{14}$ $\mathrm{g} / \mathrm{cm}^{3}$. The association of supernova explosions and neutron stars being its remnant is exemplified with the crab nebula which hosts in its center a pulsar, a rotating neutron star.

As of today more than 1600 pulsars are known and recorded in the publicly available pulsar data base at the Australian National Telescope Facility (ATNF), see [1]. The best determined mass is still the one of the HulseTaylor pulsar with $M=(1.4411 \pm 0.00035) M_{\odot}$, the fastest rotating one is the pulsar PSR J1748-2446ad with 716 revolutions per second. Recently, indications of even more massive neutron stars in Pulsar-White Dwarfs Systems have been published [2]. In detail, constraints for the masses of four pulsars with a white dwarf companion have been measured by timing the pulsar signal. For the pulsar J0751 +1807 a mass range of $M=2.1 \pm 0.2 M_{\odot}(1 \sigma$ standard deviation) and $M=1.6-2.5 M_{\odot}(2 \sigma)$ has been inferred.

Constraints on the mass and radius of neutron stars can be derived by observations in the optical as well as in the x-ray band, a booming field of exploration since the launch of the X-ray satellites Chandra and XMM-Newton in 1999. The best studied isolated neutron star is RXJ 1856.35-3754, the closest one known. A two-component blackbody fit to the combined optical and x-ray spectra results in a low soft temperature, so as not to be in contradiction with the observed x-ray flux. This low temperature implies a rather large radius, so that the optical flux comes out right. A conservative lower limit was given in
3. as $R_{\infty}=16.5 \mathrm{~km}(\mathrm{~d} / 117 \mathrm{pc})$ which would allow only for extremely stiff equations of state.

Another way of probing neutron star matter properties is by cooling observations of supernova remnants, see e.g. [4,5]. The observational limits hints at fast cooling processes in the interior of neutron stars, i.e. direct URCA reactions. Standard conventional cooling curves are too high, so that either a large nuclear asymmetry energy or strange exotic particles are needed to generate efficient and fast cooling!

The basic structure of the low-density region of neutron stars is fairly well-known. The outer crust consists of a lattice of nuclei with free electrons and is a few 100 meters thick. The sequence of nuclei is controlled by their binding energies and follows mainly along the neutron magic numbers 50 and 82 (for a most recent investigation of the outer crust see [6]). Similar features will be discussed in the context of hypernuclei below. The inner crust starts at the neutron drip density at $n=4 \cdot 10^{11}-10^{14} \mathrm{~g} / \mathrm{cm}^{3}$ and consists of a lattice of nuclei with free neutrons and electrons. The core starts at the end of the inner crust which occurs around half times normal nuclear matter density.

\section{Hyperons in Neutron Stars!}

The term neutron star implies that the main component of neutron star matter are just neutrons. However, this picture changes drastically for matter at extremely high densities, i.e. in the core of neutron stars. Simple arguments for the presence of other more exotic species besides nucleons, electrons and muons can be given in terms of a free gas of hadrons and leptons. Matter in $\beta$-equilibrium but with no interactions starts to populate $\Sigma^{-}$hyperons already at $4 n_{0}$, where $n_{0}$ is the normal nuclear matter density, the lighter $\Lambda$ hyperons appear at $8 n_{0}$ [7. Inclusion of nuclear forces generically reduces these critical densities 
Jürgen Schaffner-Bielich: Hypernuclear Physics and Compact Stars

substantially, so that hyperons appear already around $2 n_{0}$ (see e.g. [8] and references therein for the very first investigations of this kind).

That interactions are essential for the description of neutron star properties is evident from the fact that the corresponding equation of state of a free gas results in a maximum mass of only $M_{\max } \approx 0.7 M_{\odot}$ (see e.g. [9]) which is by more than a factor two smaller than the presently most precisely known pulsar mass of $1.44 M_{\odot}$ for the pulsar PSR 1913+16. Hence, effects from strong interactions are crucial in describing neutron stars raising the maximum mass from 0.7 to 2 or more solar masses [10]. Note, that this is in contrast to white dwarfs which are basically stabilised by the Fermi pressure of the free electron gas only.

As hyperons are likely to be present in addition to nucleons, one has to consider the interactions between all stable baryons. Besides the nuclear force, there is some knowledge from hypernuclear physics about the interactions between hyperons and nucleons and scarcely between hyperons themselves. The $\Lambda N$ interactions is very well studied, the potential depth of $\Lambda$ hyperons is $U_{\Lambda}=-30$ $\mathrm{MeV}$ at $n=n_{0}$ (see e.g. [11]), so that bound $\Lambda$ hypernuclear states exists. The situation is different for $\Sigma$ hyperons. The only bound $\Sigma$ hypernucleus known so far, ${ }_{\Sigma}^{4} \mathrm{He}$, is bound by isospin forces [12,13]. A detailed scan for $\Sigma$ hypernuclear states turned out to give negative results [14. The study of $\Sigma^{-}$atoms hints at a sizable repulsive potential in the nuclear core, i.e. at $n=n_{0}$. On the other hand, the $\Xi$ nucleon interactions seems to be attractive, several $\Xi$ hypernuclear states are reported in the literature [15]. More recently, quasi-free production of $\Xi$ 's reveal an attractive potential of $U_{\Xi}=-18 \mathrm{MeV}[16$, 17] (with relativistic corrections, see [18]). Last but not least, the hyperon-hyperon (YY) interaction is not really well known, there are just a few double $\Lambda$ hypernuclear events (for a recent review see [19]). The interaction between other pairs of hyperons as $\Lambda \Xi$ or $\Xi \Xi$ is not known at all experimentally. However, the hyperon potentials are essential for the determination of the composition of neutron star matter so basic hypernuclear data can provide substantial input for the modelling of neutron star matter.

Important for the stability of neutron stars is the shortrange repulsion of the baryon-baryon interaction. Fits with nonrelativistic potentials to $\Lambda$ hypernuclear data show effects from three-body interactions for the $\Lambda N$ interaction [1]. The density dependence of the Schrödinger equivalent potential is compatible with the many-body meanfield potential of relativistic field-theoretical approaches and demonstrates that the hyperon potential turns repulsive above $2 n_{0}$ [20]. The absence of these higher-order terms in density is likely to generate too soft an equation of state, so that the maximum mass of neutron stars falls below the mass limit of $1.44 M_{\odot}$. Arguably, this might be the reason that modern Brueckner calculations of neutron star matter with nucleons and hyperons result in too low neutron star maximum masses. The hyperon three-body force has not received too much attention recently, but is known for quite some time to be repulsive in nature for

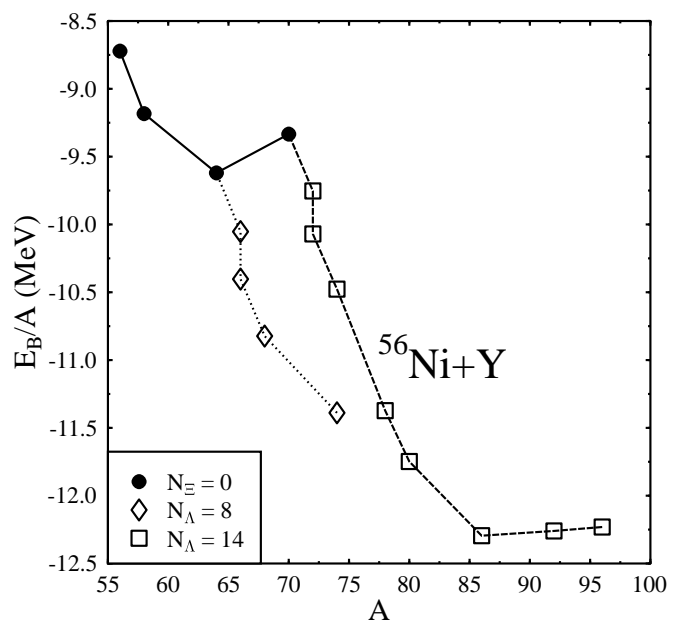

Fig. 1. The binding energy of strange hadronic matter for a nucleonic core of ${ }^{56} \mathrm{Ni}$ with added $\Lambda$ and $\Xi$ hyperons as a function of baryon number $A$ (taken from [22]).

$\Lambda N N$ [21] leading to the needed additional stability for neutron stars.

The appearance of hyperons in dense neutron star matter can be also elucidated by looking at finite systems of nucleons and hyperons, so called strange hadronic matter 23, 22, 24, 18. Let us consider an arbitrary number of nucleons and hyperons forming one big multi-hypernucleus. The system is stable against strong interactions, if reactions as $\Lambda+\Lambda \leftrightarrow \Xi+N$ and $\Sigma+N \rightarrow \Lambda+N$ are Pauliblocked. The first reaction releases an energy of $Q \approx 25$ $\mathrm{MeV}$, the second one $Q \approx 80 \mathrm{MeV}$ which hints at that $\Sigma$ hyperons can be hardly stabilised in hypernuclear systems. A similar feature will be present for neutron star matter, where it is indeed also likely that $\Sigma$ hyperons do not appear (although the main reason is due to the repulsive potential for $\Sigma$ hyperons). One can construct stable systems of nucleons and hyperons by adding successively $\Lambda$ hyperons until $\Xi$ hyperons can be populated as the filled $\Lambda$ hypernuclear levels prevent the strong reactions by Pauli-blocking. Fig. 1] shows the binding energy of such Pauli-blocked systems for a nucleonic core of ${ }^{56} \mathrm{Ni}$ versus the baryon number. When the p-shell of the $\Lambda$ hypernuclear level is filled up, $\Xi$ hyperons can be added in the s-shell without loosing stability. On the contrary, the addition of hyperons leads to an overall increase in the binding energy as the hyperons populate deep lying $\mathrm{s}^{-}$and $\mathrm{p}$ - states in a separate quantum well. The nuclear binding energy with $\Lambda$ s and $\Xi$ s reaches up to $E / A=-12 \mathrm{MeV}$ (here a weak YY interaction is assumed)! In terms of the binding energy, it is energetically favoured to add hyperons to the system. A similar effect occurs for dense matter in $\beta$-equilibrium: here beyond some critical density, the filling of low-lying (with low Fermi momenta) hyperon states in a newly opened quantum well becomes preferred compared to adding more nucleons at large Fermi momenta. Hyperons appear in dense matter when their in-medium energy equals their chemical 


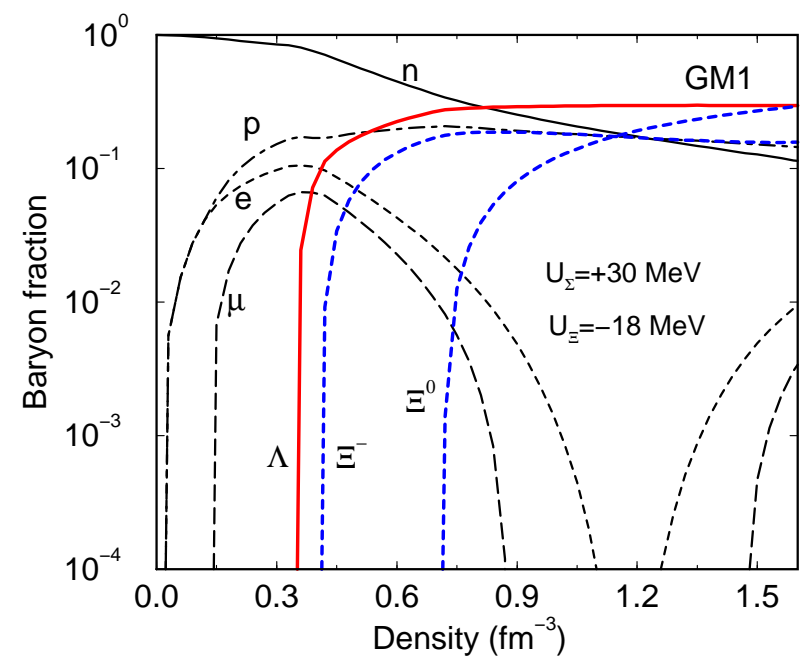

Fig. 2. The fraction of baryons and leptons in neutron star matter for a RMF calculation using set GM1 with weak hyperon-hyperon interactions (see [27]).

potential $\mu(Y)=\omega(Y)=m_{Y}+U_{Y}(n)$. Hyperons are then Pauli-blocked and can not decay as all levels are filled up for its possible decay products. In the case of neutron star matter, strange hadronic matter becomes now even stable to weak interactions!

In modern nuclear models, which are fitted to nuclear and hypernuclear data, hyperons appear in neutron star matter at $n \approx 2 n_{0}$ in relativistic mean-field (RMF) models 25, 26, 27, in a nonrelativistic potential model 28, in the quark-meson coupling model [29, in relativistic HartreeFock models [30, in Brueckner-Hartree-Fock calculations [31,32, in chiral effective Lagrangians [33, in the densitydependent hadron field theory [34] and in G-matrix calculations 35. It is remarkable that one of the very first calculations came to a similar conclusion 8 . Hence, neutron stars are indeed giant hypernuclei [25]!

The composition of neutron star matter depends sensitively on the assumed hypernuclear potentials. The $\Sigma^{-}$ hyperon appears in dense matter usually together with the $\Lambda$ at about $2 n_{0}$, in some cases even slightly before the $\Lambda$ due to its negative charge, if an attractive potential of $U_{\Sigma}=-30 \mathrm{MeV}$ similar to the $\Lambda$ is chosen. However, for a repulsive potential, the $\Sigma^{-}$as well as the other $\Sigma$ hyperons will not be present in neutron star matter at all. Fig. 2 depicts the fraction of baryons and leptons as a function of density for a relativistic mean-field calculation using the parameter set GM1 36] assuming a repulsive $\Sigma$ potential. The $\Lambda$ is present at $2.3 n_{0}$, the $\Xi^{-}$hyperon at $2.7 n_{0}$ (here the model with weak YY interaction is taken from [27]). Besides the $\Xi^{0}$ emerging at $4.7 n_{0}$ no other hyperon is present up to $10 n_{0}$, which is well beyond the maximum density reached for this equation of state. It is clear that hypernuclear data provides as an essential ingredient the hyperon potential depth which controls the composition in the core of neutron stars. The baryon and lepton population is highly sensitive to the in-medium potential of hyperons which will turn out to be important for the cooling of neutron stars.

\section{Hyperons and cooling of neutron stars}

Moderately aged neutron stars up to 1 million years after their formation will dominantly cool by volume emission of neutrinos. Cooling of photons from the surface will take over afterwards. The standard reaction for cooling is the modified URCA processes $N+p+e^{-} \rightarrow N+n+\nu_{e}$ and $N+n \rightarrow N+p+e^{-}+\bar{\nu}_{e}$ with a bystander nucleon to conserve energy and momentum. The modified URCA process is slow and leaves the neutron star quite warm until the photon cooling epoch. Much faster reactions are the direct URCA processes as $p+e^{-} \rightarrow n+\nu_{e}$ and $n \rightarrow p+e^{-}+\bar{\nu}_{e}$. However, this reaction can only proceed if the Fermi momenta fulfil the condition $p_{F}^{p}+p_{F}^{e} \geq p_{F}^{n}$. Charge neutrality implies $n_{p}=n_{e}$ or $p_{F}^{p}=p_{F}^{e}$, so that $2 p_{F}^{p}=p_{F}^{n}$. Hence, the proton fraction has to exceed $n_{p} / n \geq 1 / 9 \approx 11 \%$ for the direct nucleon URCA process to start. Relativistic calculations usually reach this value quite easily [37. From Fig. 2 one can read off the critical density for the direct nucleon URCA process to be $1.5 n_{0}$. Nonrelativistic calculations do not get that large proton fraction, as the asymmetry energy does not have the same strong density dependence as in relativistic models. In addition, nucleons are pairing strongly, so that energy is needed to break them up (recent reviews on cooling of neutron stars can be found in 38,39 ).

On the other hand, hyperons can help substantially to cool a neutron star via the hyperon direct URCA processes as $\Lambda \rightarrow p+e^{-}+\bar{\nu}_{e}$ or $\Sigma^{-} \rightarrow \Lambda+e^{-}+\bar{\nu}_{e}$. Remarkably, the hyperon direct URCA process happen immediately when hyperons are present and can also occur if there is no direct URCA process for nucleons allowed [40! There is no minimum fraction of hyperons needed, as there is no additional constraint from the charge neutrality condition as for nucleons (in reality the presence of muons gives a small critical fraction of a few per mille, see [40]). Hence, if nucleons are gapped the most important cooling mechanism involves hyperons.

For weak YY coupling or interaction strengths, there will be rapid cooling due to the presence of hyperons mimicking some more exotic agent as kaon condensation or quark matter in the core. The rapid cooling process can start basically as soon as hyperons are part of the composition of neutron star matter, which implies that there is some critical neutron star mass for fast cooling. Hyperon cooling is only suppressed by hyperon pairing gaps which are presumably much smaller than the ones for nucleons. Hence, a detailed modelling of the cooling of neutron stars demands to have a knowledge not only on the composition, which is fixed by the in-medium potential of hyperons, but also on the YY interaction strength which determines the hyperon gap energy. There exist a few studies on hyperon cooling in the literature (see [41,42, 43, 44 and references therein). In the first hyperon cooling calculation with hyperon pairing [1], hyperons are present in the core for $M \geq 1.35 M_{\odot}$. The $\Sigma^{-}$appears before the 
Jürgen Schaffner-Bielich: Hypernuclear Physics and Compact Stars

$\Lambda$ so that the dominant cooling process involves the reaction $\Sigma^{-} \rightarrow \Lambda+e^{-}+\bar{\nu}_{e}$. Two-body YY interactions were used as input to model the hyperon pairing gaps and their emissivities. It was found that hyperon gaps improve the thermal history and are more consistent with x-ray observations of neutron stars. On the other hand, in a subsequent study 42 the $\Lambda$ hyperon appeared at a slightly lower density than the $\Sigma^{-}$, so that there was a tiny density range of unpaired $\Lambda$ hyperons present. These unpaired hyperons resulted in even faster cooling for heavier stars via the hyperon direct URCA. The conclusion is, that indeed two-body forces between hyperons and nucleons have an enormous impact on the cooling history of neutron stars. Hence, hypernuclear physics serves as a key ingredient not only for the composition of dense neutron star matter but also for the cooling history of neutron stars.

\section{Hyperons and the maximum mass of neutron stars}

It is known for quite some time, that hyperons have a significant effect on the global properties of compact stars. As new degree of freedom, which can populate new Fermi levels, hyperons can lower the overall Fermi energy and momentum of baryons and leptons. Thereby, the total pressure of the system for a given energy density is considerably lowered, which implies that the equation of state is substantially softened.

The first consistent implementation of relativistic hyperon potential depths in neutron star matter was performed by Glendenning and Moszkowski [36. Using a relativistic field theoretical approach, the neutron star with nucleons and leptons only reached a maximum mass of $M \approx 2.3 M_{\odot}$. A substantial decrease of the maximum mass occurred once hyperons were taken into account, with parameters fixed by hypernuclear data. The maximum mass for such "giant hypernuclei" turned out to be now around $M \approx 1.7 M_{\odot}$. Moreover, they demonstrated that the case of noninteracting hyperons results in a too low maximum mass, i.e. $M<1.4 M_{\odot}$ ! Clearly, strong (repulsive) interactions between hyperons have to be implemented for a consistent description of pulsar masses.

The issue of the softness of the nuclear equation of state and the maximum mass of neutron stars has received considerable renewed interest recently due to the analysis of heavy-ion data. The focus will be here, in the interest of the present conference, on the analysis of strange particle production in heavy-ion collisions, in particular the subthreshold production of kaons measured by the KaoS collaboration [45] at GSI, Darmstadt. The analysis of the combined data with transport models at various collision energies and colliding systems comes to the conclusion that the nuclear equation of state should be rather soft at densities around $2-3 n_{0}$ 46. The important point is that this conclusion is insensitive to the underlying microphysical input for the transport simulation, as the kaonnucleon optical potential, cross sections, lifetime of resonances etc. The extracted compression modulus turns out to be around $200 \mathrm{MeV}$ for a simple Skyrme-type parameterisation of the nuclear equation of state.

However, as outlined above, most recent pulsar data points towards quite large masses which can be only reconciled with a very stiff hadronic equation of state. There seems to be an obvious conflict between heavy-ion data and pulsar observations. The apparent contradiction can be resolved by noting that transport models use actually the Schrödinger equivalent potential as input not the nuclear equation of state. Second, the nuclear density ranges probed are different for the production of kaons and the maximum mass of neutron stars. Typically, the maximum central density reached in the center of neutron stars amounts to about say $5-6 n_{0}$, which of course depends on the assumed hadronic model. These values could be much larger. However, one hardly finds a calculation in the literature with substantially lower values for the maximum central densities. As stated above, kaon production in heavy-ion collisions is sensitive to $2-3 n_{0}$. Therefore, there is a gap in the nuclear density regions probed. The stiffness of the hadronic equation of state above $2-3 n_{0}$ controls the value of the maximum mass achievable for neutron stars. Interestingly, this is the density regime where hyperons presumably appear and modify the neutron star matter properties significantly. These lines of arguments have been cross-checked in a more detailed investigation using Skyrme-type and relativistic mean-field models which will be reported elsewhere [4]. The 'soft nuclear equation of state' extracted from heavy-ion data is indeed compatible with the recent pulsar mass measurements when only nucleons and leptons are considered as the basic constituents in neutron star matter. The inclusion of hyperons, however, causes an equation of state which turns to be too soft at high densities with the constraint from heavy-ion data, so that the maximum mass is lower than the limit from pulsar data!

Again, hyperons play a decisive role in compact star physics. The feature, that hyperons lower drastically the maximum mass of neutron stars became even more pronounced with modern many-body approaches to neutron star matter beyond the mean-field approximation. In relativistic Hartree-Fock calculations, the maximum mass of neutron stars was computed to be $M_{\max }=1.4-1.8 M_{\odot}$ depending sensitively on the chosen hyperon coupling strength 48. In Brueckner-Hartree-Fock approaches using Nijmegen soft-core hyperon-nucleon (YN) potentials maximum masses of $M_{\max }=1.47 M_{\odot}$ have been derived for the nucleonnucleon and $\mathrm{YN}$ interactions only and $M_{\max }=1.34 M_{\odot}$ when including the YY interactions [49]. In the same approach, three-body forces for nucleons have been included but none for the hyperons so that a maximum mass of only $M_{\max }=1.26 M_{\odot}$ was attained 50. All these mass limits are below the new mass limit of $1.6 M_{\odot}$ for the pulsar J0751+1807, the hyperonic equations of state are just too soft. Clearly, some additional hyperon physics is missing. Presumably, three-body force for hyperons will solve this problem, as it is repulsive and will raise the maximum mass (some crude investigations in this directions can be found in 35] supporting this statement). Here, 
input is needed from hypernuclear physics, not only for the hyperon three-body force but also for the momentum dependence of the hyperon interactions, as dense matter probes momenta of the order of several hundred MeVs. Contrary to the widely used standard mean-field and nonrelativistic approaches, Brueckner-type approaches adopt momentum-dependent potentials which have to be fixed by $\mathrm{YN}$ scattering and hypernuclear data.

The YY interaction is another important ingredient for the description of neutron star matter. In fact, it is even possible to generate a new class of compact stars, hyperon stars, besides ordinary white dwarfs and neutron stars, by a newly emerging stable solution of the Tolman-Oppenheimer-Volkoff equation [51. By increasing the overall strength of the YY interactions (in particular the unknown $\Xi \Xi$ interaction which can be probed in heavy-ion collisions however, see [52]), a first order phase transition appears from neutron matter to hyperon-rich matter. A mixed phase is present for a wide range of densities $n_{\text {mix }}=(2.5-6.5) n_{0}$. Interestingly, all hyperons $(\Lambda$, $\Xi^{0}, \Xi^{-}$) appear at the start of the mixed phase, as the bubbles of the new hyperon phase are charged and have a larger density than the surrounding normal neutron matter (note that for a Gibbs construction the chemical potentials must be equal in phase equilibrium, not the densities). The strong first order phase transition due to hyperons has a strong impact on the mass-radius relation for compact stars. A new stable solution in the mass-radius diagram appears, as the curve reaches a second maximum for the mass for small radii. Those hyperon stars are generated via attractive YY interactions (mainly $\Xi \Xi$ interactions) compatible with presently available hypernuclear data. We note that a weak $\Lambda \Lambda$ does not rule out a strong $\Xi \Xi$ interaction nor the possible existence of hyperon stars. The two different solutions behave like neutron star twins: they have similar maximum masses, $M_{\text {hyp }} \sim M_{n}$, but different radii $R_{\text {hyp }}<R_{n}$. In addition, selfbound compact stars for strong YY attraction with $R=7-8 \mathrm{~km}$ are also possible, but demand that strange hadronic matter is absolutely stable so that ordinary neutron stars are completely converted to hyperon stars.

Such neutron star twin solutions have been also found for a strong first order phase transition to quark matter [53,54,55. In fact, any strong first order phase transition can produce a so-called third family of compact stars. Signals for a such a strong phase transition can in principle be derived by direct mass and radius measurements, or by the collapse of a neutron star to the third family via measurements of gravitational waves, $\gamma$-rays, and neutrinos.

Redshifted spectral lines measured have been claimed to be extracted from the analysis of x-ray bursts from EXO 0748-676 [56], which give a constraint on the massradius ratio of the compact star. A recent analysis of Özel comes to the conclusion that the compact star mass is $M \geq 2.10 \pm 0.28 M_{\odot}$ with a radius of $R \geq 13.8 \pm 1.8 \mathrm{~km}[57]$ claiming that 'unconfined quarks do not exist at the center of neutron stars'! However, this conclusion was put into perspective in a follow-up reply [58] which demonstrated that those limits rule out a soft equations of state, but not quark stars or hybrid stars. The interactions between quarks can be strongly repulsive so that the presence of quark matter in the core stabilise the compact star. On the other hand, the mass limit provides indeed a strong constraint for hyperons in dense neutron star matter. Hyperons are likely to appear at moderate densities, which will substantially decrease the maximum mass. This conclusions is guided by hypernuclear data and present model calculations. If such massive neutron stars are confirmed in the future, say with masses above $2 M_{\odot}$, then it seems that our present understanding of hypernuclear physics of compact stars will be in conflict with pulsar data!

In passing, I note that strange multiquark states can also exist in neutron stars, as the H-dibaryon [59] or strange pentaquarks 60. Pentaquarks in neutron star matter will further reduce the maximum mass, which is being sensitive to the $\Theta^{+}$potential. The pentaquark $\Theta^{+}$appears around $4 n_{0}$ for a potential depth of $U\left(\Theta^{+}\right)=-100 \mathrm{MeV}$ at $n_{0}$. For the maximum mass star the $\Theta^{+}$population amounts to $5 \%$ in the core. Present pulsar mass limits, however, do provide a very weak constraint on $\Theta^{+}$potential (e.g. for $M>1.6 M_{\odot}$, the potential depth should $U\left(\Theta^{+}\right)>-190$ $\mathrm{MeV}$ ) which are a much stronger for a hypothetical negatively charged $\Theta^{-}$.

\section{Summary}

As outlined above, that hyperons have a substantial impact on neutron star properties. There is a sizable decrease in the maximum mass of neutron stars due to the presence of hyperons in the core. The $\Lambda$ hyperons appear at $n \approx 2 n_{0}$ in neutron star matter. The population of $\Sigma$ hyperons hinges crucially on their in-medium potential. They are likely to be absent for a repulsive potential, but the negatively charged $\Sigma^{-}$could be the first exotic component in neutron star matter for an attractive potential. A tiny amount of hyperons can suffice to cool neutron stars rapidly by the hyperon direct URCA process, which is controlled by hyperon pairing gaps. A strongly attractive YY interaction, between $\Xi$ hyperons, results in a first order phase transition from neutron-rich to hyperon-rich matter. This transition allows for a new, stable solution for compact stars, hyperon stars, with similar masses but smaller radii. Pulsar mass measurements can give constraints on multiquark states in dense matter, e.g. for hyponuclei, nuclear systems with a bound pentaquark state.

It is obvious, that hypernuclear physics provides essential input for compact star physics. The YN interactions, in particular the potential depth in bulk nuclear matter, controls the population of hyperons for massive neutron stars, the first exotic component likely to appear for supranuclear densities present in the core. The emergence of hyperons softens the nuclear equation of state and the maximum neutron star mass possible considerably which depends on the YN coupling strength and sensitively on the hyperon three-body forces. Two-body YY interactions regulate the cooling behaviour of massive neutron stars, as the hyperon direct URCA reaction is suppressed by hyperon gaps. In addition, hyperons can 
generate a new class of compact stars, hyperon stars, for a suitably attractive YY potential. The ongoing and future experimental hypernuclear programs (see these proceedings) at DA $\Phi$ NE, Jefferson Lab, KEK, J-PARC, MAMI$\mathrm{C}$, and at GSI, Darmstadt, in particular the HypHI program and HYPER-GAMMA with PANDA at FAIR, will provide here the decisive inputs for addressing the global features as well as the cooling properties of neutron stars.

\section{References}

1. http://www.atnf.csiro.au/research/pulsar/psrcat/.

2. D. J. Nice, E. M. Splaver, I. H. Stairs, O. Löhmer, A. Jessner, M. Kramer, and J. M. Cordes, Astrophys. J. 634, 1242 (2005), astro-ph/0508050.

3. J. E. Trümper, V. Burwitz, F. Haberl, and V. E. Zavlin, Nucl. Phys. Proc. Suppl. 132, 560 (2004), astro$\mathrm{ph} / 0312600$.

4. D. L. Kaplan, D. A. Frail, B. M. Gaensler, E. V. Gotthelf, S. R. Kulkarni, P. O. Slane, and A. Nechita, Astrophys. J. Suppl. 153, 269 (2004), astro-ph/0403313.

5. D. L. Kaplan, B. M. Gaensler, S. R. Kulkarni, and P. O. Slane, Astrophys. J. Suppl. 163, 344 (2006), astro$\mathrm{ph} / 0602312$.

6. S. B. Rüster, M. Hempel, and J. Schaffner-Bielich, Phys. Rev. C 73, 035804 (2006), astro-ph/0509325.

7. V. A. Ambartsumyan and G. S. Saakyan, Sov. Astron. 4, 187 (1960).

8. G. S. Sahakian and Y. L. Vartanian, Nuovo Cimento 30, $82(1963)$.

9. J. R. Oppenheimer and G. M. Volkoff, Phys. Rev. 55, 374 (1939).

10. A. G. W. Cameron, Astrophys. J. 130, 884 (1959).

11. D. J. Millener, C. B. Dover, and A. Gal, Phys. Rev. C 38, 2700 (1988).

12. R. S. Hayano et al., Phys. Lett. B 231, 355 (1989).

13. T. Nagae et al., Phys. Rev. Lett. 80, 1605 (1998).

14. S. Bart et al., Phys. Rev. Lett. 83, 5238 (1999).

15. C. B. Dover and A. Gal, Ann. Phys. (N.Y.) 146, 309 (1983).

16. T. Fukuda et al. (E224), Phys. Rev. C 58, 1306 (1998).

17. P. Khaustov et al. (AGS E885), Phys. Rev. C 61, 054603 (2000).

18. J. Schaffner-Bielich and A. Gal, Phys. Rev. C 62, 034311 (2000).

19. A. Gal, Nucl. Phys. A754, 91 (2005), nucl-th/0312071.

20. J. Schaffner-Bielich, I. N. Mishustin, and J. Bondorf, Nucl. Phys. A625, 325 (1997).

21. A. Gal, Phys. Rev. Lett. 18, 568 (1967).

22. J. Schaffner, C. B. Dover, A. Gal, C. Greiner, and H. Stöcker, Phys. Rev. Lett. 71, 1328 (1993).

23. J. Schaffner, C. Greiner, and H. Stöcker, Phys. Rev. C 46, 322 (1992).

24. J. Schaffner, C. B. Dover, A. Gal, D. J. Millener, C. Greiner, and H. Stöcker, Ann. Phys. (N.Y.) 235, 35 (1994).

25. N. K. Glendenning, Astrophys. J. Lett. 293, 470 (1985).

26. R. Knorren, M. Prakash, and P. J. Ellis, Phys. Rev. C 52, 3470 (1995).

27. J. Schaffner and I. N. Mishustin, Phys. Rev. C 53, 1416 (1996).
28. S. Balberg and A. Gal, Nucl. Phys. A625, 435 (1997).

29. S. Pal, M. Hanauske, I. Zakout, H. Stöcker, and W. Greiner, Phys. Rev. C 60, 015802 (1999).

30. H. Huber, F. Weber, M. K. Weigel, and C. Schaab, Int. J. Mod. Phys. E7, 301 (1998).

31. M. Baldo, G. F. Burgio, and H. J. Schulze, Phys. Rev. C 61, 055801 (2000).

32. I. Vidana, A. Polls, A. Ramos, L. Engvik, and M. HjorthJensen, Phys. Rev. C 62, 035801 (2000).

33. M. Hanauske, D. Zschiesche, S. Pal, S. Schramm, H. Stöcker, and W. Greiner, Astrophys. J. 537, 958 (2000).

34. F. Hofmann, C. M. Keil, and H. Lenske, Phys. Rev. C 64, 025804 (2001), nucl-th/0008038.

35. S. Nishizaki, T. Takatsuka, and Y. Yamamoto, Prog. Theor. Phys. 108, 703 (2002).

36. N. K. Glendenning and S. A. Moszkowski, Phys. Rev. Lett. 67, 2414 (1991).

37. J. M. Lattimer, M. Prakash, C. J. Pethick, and P. Haensel, Phys. Rev. Lett. 66, 2701 (1991).

38. D. G. Yakovlev and C. J. Pethick, Ann. Rev. Astron. Astrophys. 42, 169 (2004), astro-ph/0402143.

39. A. Sedrakian, Prog. Part. Nucl. Phys. 58, 168 (2007), nuclth/0601086.

40. M. Prakash, M. Prakash, J. M. Lattimer, and C. J. Pethick, Astrophys. J. Lett. 390, L77 (1992).

41. C. Schaab, S. Balberg, and J. Schaffner-Bielich, Astrophys. J. Lett. 504, L99+ (1998), astro-ph/9807198.

42. D. Page, M. Prakash, J. M. Lattimer, and A. Steiner, Phys. Rev. Lett. 85, 2048 (2000).

43. I. Vidaña and L. Tolós, Phys. Rev. C 70, 028802 (2004), nucl-th/0405010.

44. T. Takatsuka, S. Nishizaki, Y. Yamamoto, and R. Tamagaki, Prog. Theor. Phys. 115, 355 (2006), nucl-th/0601043.

45. A. Forster et al., Phys. Rev. C 75, 024906 (2007), nuclex/0701014.

46. C. Hartnack, H. Oeschler, and J. Aichelin, Phys. Rev. Lett. 96, 012302 (2006), nucl-th/0506087.

47. in preparation.

48. H. Huber, M. K. Weigel, and F. Weber, Z. Naturforsch. 54A, 77 (1999), astro-ph/9811463.

49. I. Vidana, A. Polls, A. Ramos, L. Engvik, and M. HjorthJensen, Phys. Rev. C 62, 035801 (2000), nucl-th/0004031.

50. M. Baldo, G. F. Burgio, and H. J. Schulze, Phys. Rev. C 61, 055801 (2000), nucl-th/9912066.

51. J. Schaffner-Bielich, M. Hanauske, H. Stöcker, and W. Greiner, Phys. Rev. Lett. 89, 171101 (2002), astroph/0005490.

52. J. Schaffner-Bielich, R. Mattiello, and H. Sorge, Phys. Rev. Lett. 84, 4305 (2000).

53. N. K. Glendenning and C. Kettner, Astron. Astrophys. 353, L9 (2000), astro-ph/9807155.

54. K. Schertler, C. Greiner, J. Schaffner-Bielich, and M. H. Thoma, Nucl. Phys. A677, 463 (2000), astro-ph/0001467.

55. E. S. Fraga, R. D. Pisarski, and J. Schaffner-Bielich, Phys. Rev. D 63, 121702(R) (2001), hep-ph/0101143.

56. J. Cottam, F. Paerels, and M. Mendez, Nature 420, 51 (2002), astro-ph/0211126.

57. F. Ozel, Nature 441, 1115 (2006), astro-ph/0605106.

58. M. Alford, D. Blaschke, A. Drago, T. Klähn, G. Pagliara, and J. Schaffner-Bielich, Nature 445, E7 (2006), astro$\mathrm{ph} / 0606524$.

59. N. K. Glendenning and J. Schaffner-Bielich, Phys. Rev. C 58, 1298 (1998).

60. I. Sagert, M. Wietoska, and J. Schaffner-Bielich, J. Phys. G 32, S241 (2006), astro-ph/0608317. 